\title{
Gastric Composite Tumor: A Mixed Adenocarcinoma and Neuro- endocrine Tumor with Goblet Cell Carcinoid Component
}

\author{
Archana Lakshmanan $^{1^{*}}$, Ann Kurian ${ }^{2}$ and Annapurneswari Subramanyan ${ }^{2}$ \\ ${ }^{1}$ Junior Consultant, Apollo Hospitals, India \\ ${ }^{2}$ Senior Consultant, Apollo Hospitals, India
}

*Corresponding author: Dr. Archana Lakshmanan, Junior Consultant, Apollo Hospitals, Chennai, India, Tel: 9144-9500066561

\begin{abstract}
Goblet cell carcinoids are distinct neoplasms with combined histologic features of carcinoid and adenocarcinoma with intermediate prognosis. They were initially thought to arise only from the appendix, however, in the recent past, a few cases have been described in extra appendiceal locations. Mixed adenoneuroendocrine tumors of the stomach are uncommon and that including Goblet cell carcinoid component is exceedingly rare. We report a case of composite tumor involving the stomach with four distinct neoplastic cell populations including Goblet cell carcinoid.
\end{abstract}

\section{Keywords}

MANEC, Goblet cell carcinoid, Neuroendocrine tumor

\section{Introduction}

Mixed adenoneuroendocrine carcinomas (MANEC) are rare malignancies and show a wide spectrum, ranging from adenocarcinomas with interspersed neuroendocrine cells to neuroendocrine tumors with focal exocrine component. They have variable degrees of differentiation, with features ranging from adenomas to adenocarcinomas and from well to poorly differentiated neuroendocrine tumors [1]. According to 2010 World Health Organization classification of tumors of digestive system, Mixed Exocrine- Neuroendocrine carcinomas were renamed as mixed adenoneuroendocrine carcinomas (MANEC) [2]. The term MANEC can be used, only when both the components are present in significant proportions (30\% of the entire tumor) [1,2].

Goblet cell carcinoids (GCC) are unique neoplasms, which has been described almost exclusively in the ap- pendix. There are few case reports of extra appendiceal goblet cell carcinoids $[3,4]$. We report a rare case of gastric composite tumor with mixed adenocarcinoma and neuroendocrine tumor with a goblet cell carcinoid component and we reviewed the literature pertaining to these tumors.

\section{Case Report}

A 72-year-old man presented with complaints of abdominal pain and melena for 6 months duration. Oesophagogastroduodenoscopy revealed a raised ulcerated lesion involving the gastric antrum with partial gastric outlet obstruction. The antral lesion was biopsied and reported elsewhere as poorly differentiated adenocarcinoma. The staging computed tomography (CT) abdomen demonstrated a thickened pylorus with enlarged perigastric lymph nodes. Other visceral organs including appendix were normal. The patient was then referred to our institution for further treatment, wherein a total gastrectomy with omentectomy and regional lymph node dissection was done. Intraoperatively, there was no evidence of distant metastases. The post-operative course was uneventful, and at the 3-month follow-up, the patient was alive and well without evidence of recurrence. The patient was then lost to follow-up.

\section{Materials and Methods}

Specimen was fixed in formalin, representative sections taken (one section per centimeter of tumor), then paraffin embedded, sectioned at 4-micron thickness and stained with hematoxylin-eosin (H\&E), Periodic

\footnotetext{
Citation: Lakshmanan A, Kurian A, Subramanyan A (2018) Gastric Composite Tumor: A Mixed Adenocarcinoma and Neuroendocrine Tumor with Goblet Cell Carcinoid Component. Int J Pathol Clin Res 4:077. doi.org/10.23937/2469-5807/1510077

Accepted: October 10, 2018: Published: October 12, 2018

Copyright: (C) 2018 Lakshmanan A, et al. This is an open-access article distributed under the terms of the Creative Commons Attribution License, which permits unrestricted use, distribution, and reproduction in any medium, provided the original author and source are credited.
} 

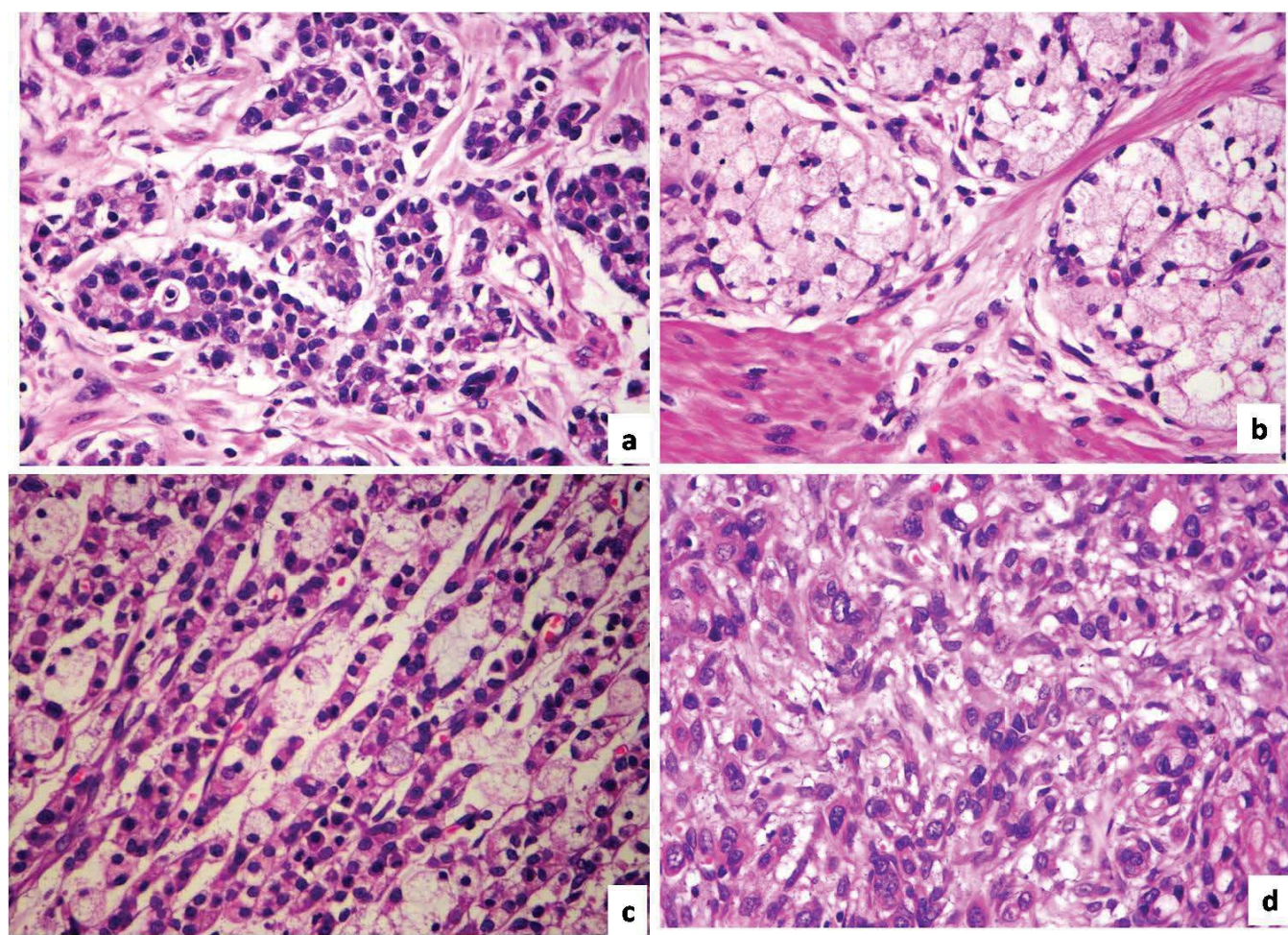

Figure 1: Four distinct histomorphologic patterns (H\&E-40X) (a) Well differentiated neuroendocrine tumor; (b) Goblet cell carcinoid; (c) Signet ring cell carcinoma; (d) Poorly differentiated adenocarcinoma.

Acid Schiff with Diastase (PASD), Mucicarmine, Grimelius and Combined Alcian blue- Periodic Acid Schiff technique (AB-PAS). Immunohistochemical staining was performed in Ventana automated immunostainer (Benchmark XT) using manufacturer's protocol.

\section{Gross findings}

Grossly there was an ulcero infiltrative lesion involving the entire circumference of the gastric antrum measuring $6.5 \times 6.0 \times 1.0 \mathrm{~cm}$. The lesion infiltrated the gastric wall transmurally involving all the layers of stomach extending focally into omental adipose tissue. Rest of the gastric mucosa was unremarkable.

\section{Light microscopic findings}

Microscopically there was extensive mucosal ulceration and infiltration by a tumor with four distinct histomorphologic patterns (Figure 1) (1) Goblet cell carcinoid; (2) Well differentiated neuroendocrine tumor; (3) Signet ring cell carcinoma; (4) Conventional poorly differentiated adenocarcinoma. Goblet cell carcinoid was the predominant component, seen mainly in the submucosa and muscularis propria as small nests and rosettes of cells with abundant mucin filled clear cytoplasm and eccentrically placed nuclei. Rest of the components were noted focally. Signet ring cell carcinoma was morphologically similar to that of goblet cell carcinoid, but the cells were predominantly seen within the mucosa as sheets and the cells exhibited nuclear atypia. The well differentiated neuroendocrine tumor was seen focally within the submucosa and muscularis propria as monotonous population of cells with moderate eosinophilic granular cytoplasm and round nuclei with stippled chro- matin arranged as nests, trabeculae and tubules. Focal areas showed a poorly differentiated adenocarcinoma component, comprising of atypical cells with moderate to marked nuclear atypia arranged as nests and cords with rare glands. These four components were noted as intermingled with each other at many foci. Perineural and lymphovascular invasion were noted. Adjacent gastric mucosa showed focal intestinal metaplasia. There was no evidence of atrophy, activity, Helicobacter pylo$\mathrm{ri}$, neuroendocrine hyperplasia or dysplasia in the rest of the gastric mucosa. Metastatic carcinoma was noted in the lymph nodes which morphologically resembled an adenocarcinoma.

The goblet cell carcinoid, signet ring cell carcinoma and conventional adenocarcinoma components were variably positive for mucin (PASD, Mucicarmine and ABPAS). Grimelius was positive focally in both goblet cell carcinoid and well differentiated neuroendocrine tumor and was negative in the other two components.

\section{Immunohistochemistry findings}

Immunohistochemistry results are mentioned in Figure 2. All the tumor components displayed an intense membranous staining for Cytokeratin. Synaptophysin and Chromogranin were strongly positive in well differentiated neuroendocrine tumor, variably positive in goblet cell carcinoid areas and absent in poorly differentiated adenocarcinoma and signet ring cell carcinoma areas. Carcinoembryonic antigen (CEA) was variably positive in all the four components. E-cadherin immunostain showed a strong membranous staining in classic Neuroendocrine tumor where as it was weakly positive 


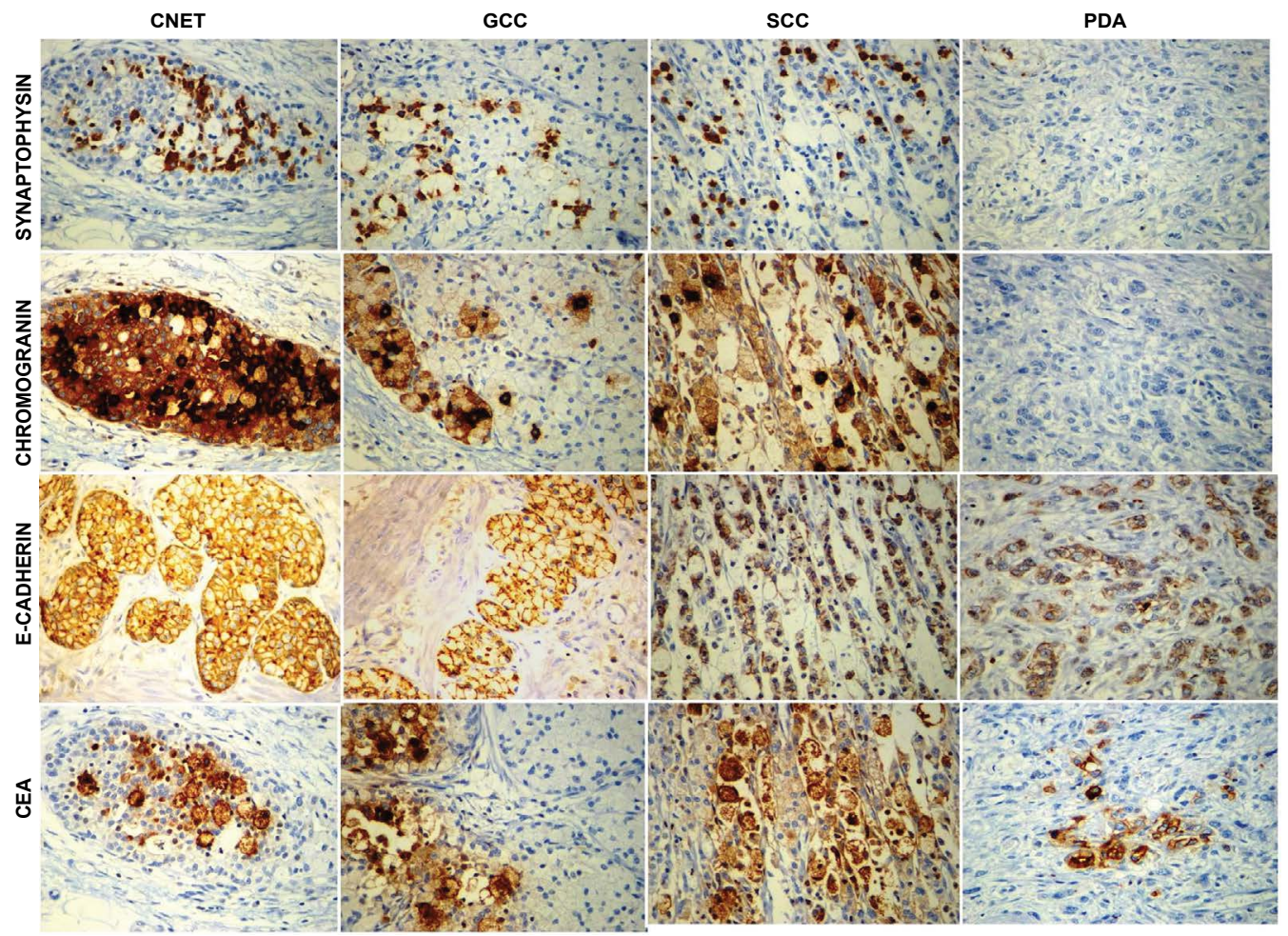

Figure 2: Synaptophysin and chromogranin were positive in well differentiated neuroendocrine tumour and goblet cell carcinoid and negative in signet ring cell and conventional adenocarcinoma. CEA is variably positive in all the four components. E Cadherin is strongly positive in classic and goblet cell carcinoid, where as it was weak in signet ring cell and conventional adenocarcinoma.

in goblet cell carcinoid, poorly differentiated adenocarcinoma and signet ring cell carcinoma areas.

\section{Discussion}

Goblet cell carcinoids arising at extra appendiceal locations although extremely rare, do exist which was confirmed by a study conducted by Gui X, et al. on extra appendiceal goblet cell carcinoids arising from various locations in the gastrointestinal tract [3].

To date a few cases of mixed adenoneuroendocrine tumor with well differentiated neuroendocrine tumor component have been reported in the literature [5-7], however, tumors with goblet cell carcinoid component in the stomach has not been well delineated. There are few case reports similar to the present case. Fujiyoshi, et al. reported two cases of composite glandular endocrine cell carcinoma of the stomach with morphology similar to the present case with four similar distinct neoplastic populations [8]. Caruso, et al. also reported a case with four distinct components comprising of mucinous adenocarcinoma, glandular adenocarcinoma, carcinoid and goblet cell carcinoid [9]. As described in their case report by Fujiyoshi, et al. [8], E cadherin was found to be helpful in delineating the goblet cell carcinoid from signet ring cell carcinoma component as evident by a strong membranous staining pattern in GCC and weak staining in Signet ring cell carcinoma areas.

Though there are no well-defined classification of GCC arising from stomach, Tang, et al. classified the GCC of appendix into three groups with typical GCC (Group
A) and adenocarcinoma exGCC further divided into adenocarcinoma exGCC, signet ring cell type (Group B) and adenocarcinoma exGCC, poorly differentiated adenocarcinoma type (Group C) [10]. The present case with poorly differentiated adenocarcinoma component would fall into Group C.

Tang, et al. [10] and Taggart, et al. [11] found that the amount of adenocarcinoma component predicted the clinical behaviour and the overall prognosis of the patients of Adenocarcinoma exGCC involving appendix. Due to their rarity, our knowledge of behaviour and prognosis of gastric MANECs with goblet cell carcinoid is limited and mainly based on a small series of patients and case reports. The clinical behaviour of the reported gastric MANECs with GCC component were generally good with a better clinical outcome [8,9]. Thus, it is important to differentiate a Goblet cell carcinoid component from signet ring cell carcinoma and to recognize it as a component of MANECs, in determining the prognosis.

\section{Conflict of Interest}

None.

\section{Source of Support}

None.

\section{References}

1. La Rosa S, Marando A, Sessa F, Capella C (2012) Mixed adenoneuroendocrine carcinomas (MANECs) of the gastrointestinal tract: An update. Cancers (Basel) 4: 11-30. 
2. Bosman FT, Carneiro F, Hruban RH, Theise ND (2010) WHO Classification of tumours of the digestive system, fourth edition. IARC, Lyon, France, 13-14.

3. X Gui, L Qin, ZH Gao, V Falck, N Harpaz (2011) Goblet cell carcinoids at extra appendiceal locations of gastrointestinal tract: an underrecognized diagnostic pitfall. J Surg Onco 103: 790-795.

4. PH Watson, A Alguacil-Garcia (1987) Mixed crypt cell carcinoma. A clinicopathological study of the so-called 'goblet cell carcinoid'. Virchows Arch A Pathol Anat Histopathol 412: 175-182.

5. Ünal B, Elpek GO, Gelen T, Gürkan A, Yildirim B (2013) Gastric collision tumor: Case report of a rare adenocarcinoma and a typical carcinoid tumor. Oncol Lett 6: 212-214.

6. Morishita Y, Sugitani M, Sheikh A, Nemoto N, Fujii M, et al. (2005) Collision tumor of the stomach: a rare case of an adenocarcinoma and carcinoid tumor. Arch Pathol Lab Med 129: $407-409$.

7. Adhikari D, Conte C, Eskreis D, Urmacher C, Ellen K (2002)
Combined adenocarcinoma and carcinoid tumor in atrophic gastritis. Ann Clin Lab Sci 32: 422-427.

8. Fujiyoshi $\mathrm{Y}$, Kuhara H, Eimoto T (2005) Composite glandular-endocrine cell carcinoma of the stomach. Report of two cases with goblet cell carcinoid component. Pathol Res Pract 200: 823-829.

9. RA Caruso, MF Heyman, L Rigoli, C Inferrera (1998) Composite early carcinoma (ordinary adenocarcinoma carcinoid, microglandular-goblet cell carcinoid, neuroendocrine mucinous carcinoma) of the stomach. Histopathology 32: 569-571.

10. LH Tang, J Shia, RA Soslow, Dhall D, Wong WD, et al. (2008) Pathologic classification and clinical behavior of the spectrum of goblet cell carcinoid tumors of the appendix. Am J Surg Pathol 32: 1429-1443.

11. Taggart MW, Abraham SC, Overman MJ, Mansfield PF, Rashid A (2015) Goblet cell carcinoid tumor, mixed goblet cell carcinoid-adenocarcinoma, and adenocarcinoma of the appendix: Comparison of clinicopathologic features and prognosis. Arch Pathol Lab Med 139: 782-790. 